\title{
Numerical Simulation for Air Flow in the Mini-Environment and SMIF Enclosure
}

\author{
Huan-Ruei Shiu, Hsiao-Yi Huang, Sih-Li Chen, and Ming-Tsun Ke
}

\begin{abstract}
The application of mini-environment and standard mechanical interface (SMIF) enclosure in the clean room can efficiently reduce airborne particles and isolate the personnel from the product. The purpose of this article is to reduce the recirculation zone and to maintain the positive pressure from the analysis results of the airflow field and pressure distribution of SMIF enclosure and mini-environment. The simulation code CFX will be used to study the flow field of air movement corresponding to the associated design parameters. The results show that proper drilling holes or slots can reduce the circulation zones of SMIF enclosure. The positive pressure of SMIF enclosure is mainly affected by inlet air flux, area of outlets, and leakage area. The calculated results can provide the design rules for SMIF Robot inside the SMIF enclosure and reduce the particle accumulation during robot moving.
\end{abstract}

Index Terms-Air cleanliness, air flow field, mini-environment, SMIF enclosure.

$C_{1}, C_{2}, C_{3}$
$\Gamma_{i j}$
$u_{i j}$
$S_{\varphi}$
$\xi_{m}$
$\varphi$
$u, v, w$
$\rho$
$\varepsilon$
$\kappa$
$\sigma_{k}, \sigma_{\epsilon}$

Turbulent model constant.

Effective diffusive coefficient.

Velocity vector.

Source or sink.

Residual of the mass flow rate.

General fluid property variable.

Velocity component.

Density.

Turbulent dissipation.

Turbulent kinetic energy.

Turbulent Prandtl-Schmidt number.

\section{INTRODUCTION}

A $S$ THE SEMICONDUCTOR process technologies advance in leaps and bounds, the manufacturing operations take place at almost the molecular level. The requirement on the cleanliness of the process environments has increased as a result. However, conventional clean rooms no longer meet the critical demand on air quality, thereby giving rise to the design concepts of mini-environments. Hewlett-Packard Laboratories first proposed the concept of using standard mechanical interface (SMIF) wafer cassettes for transport in the manufacturing of very large integrated circuit [1]. The main advantage of mini-environment and SMIF enclosure is the reduction of the area requiring high degree of cleanliness, which decreases the construction cost and operating cost of a

Manuscript received November 8, 2001; revised August 8, 2002.

H.-R. Shiu, H.-Y. Huang and S.-L. Chen are with the Institute of Mechanical Engineering, National Taiwan University, Taiwan, R.O.C.

M.-T. Ke is with the Department of Air-Conditioning and Refrigeration Engineering, National Taipei University of Technology, Taiwan, R.O.C. (e-mail: mtke@ntut.edu.tw).

Digital Object Identifier 10.1109/TSM.2002.807737

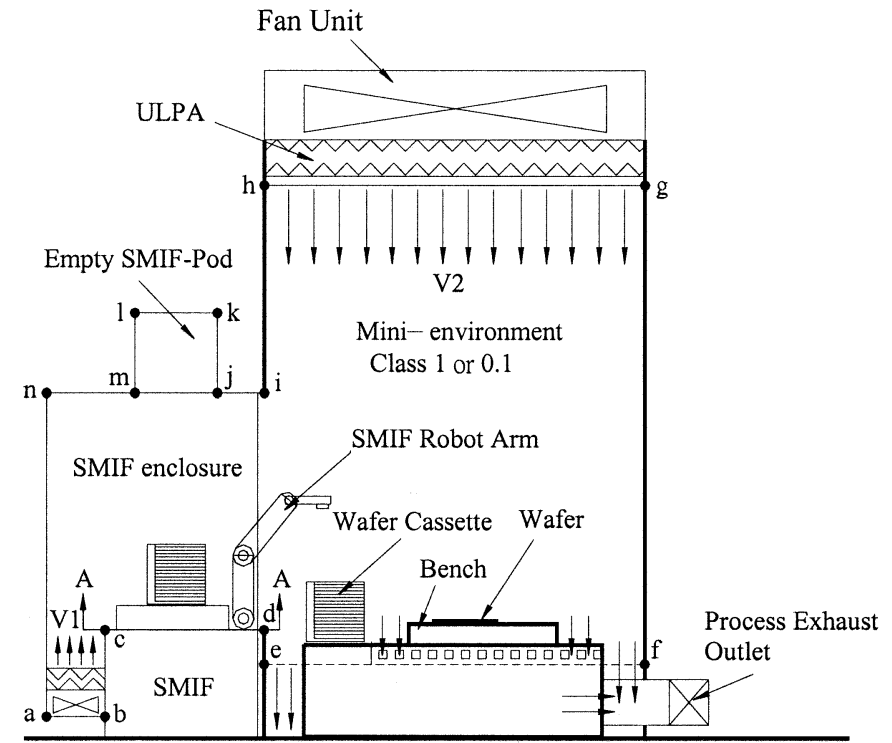

Fig. 1. Schematic diagram of SMIF enclosure and mini-environment.

clean room substantially. The effective isolation of the product from the major pollution source also stabilizes the control of cleanliness, significantly increases the product yield. Besides, it can also isolate special equipment or the equipment containing toxic chemicals, thereby reducing the health risks to personnel and providing operators with a more comfortable environment. The primary disadvantage is the greater complexity in fab. design, which requires more equipment accessories and slows down the process. In addition, the multiple environment isolation design naturally adds to the inconvenience of product transport and easily causes cross contamination. To improve the flexibility and efficiency of the application of mini-environments, the application of self-cleanliness system to avoid cross contamination in SMIF enclosure now holds the key to the development of mini-environments. Many engineers and researchers have conducted numerical simulation and experimental measurement studies related to the control of cleanliness in mini-environments or to propose the design rules of combining SMIF with mini-environments [2]-[7].

This paper presents the numerical simulation to analyze a SMIF enclosure with the objective of providing the rules for the air purification design in wafer isolation and automatic transport equipment. Fig. 1 indicates the front view of SMIF enclosure and mini-environment. The present physical model considers a clean domain combining SMIF enclosure and mini-environment. The SMIF enclosure includes an environment that contains a clean air supply system with velocity $v_{1}$ inside the 


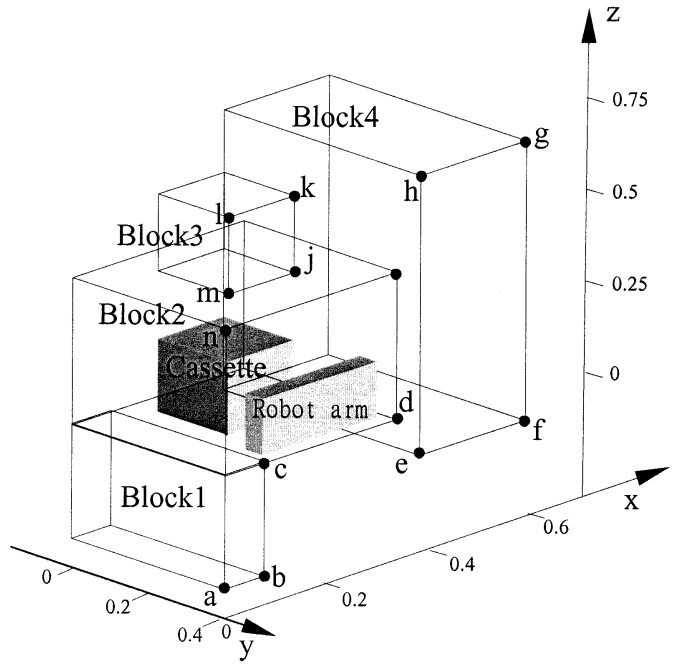

Fig. 2. Illustration of geometry model of SMIF enclosure and minienvironment.

TABLE I

GRIDS FOR COMPUTING DOMAINS

\begin{tabular}{llll}
\hline Domains & Illustrations & Block Sizes $(\mathrm{mm})$ & Number of cells \\
\hline Block-1 & Inlet chamber & $115^{*} 325^{*} 270$ & $16^{*} 30^{*} 8$ \\
\hline Block-2 & SMIF enclosure & $490^{\star} 450^{*} 470$ & $72^{\star} 40^{*} 41$ \\
\hline Block-3 & Empty SMIF Pod & $290^{*} 280^{*} 210$ & $44^{*} 24^{*} 8$ \\
\hline Block-4 & Mini-environment & $300^{*} 570^{*} 1000$ & $10^{*} 50^{*} 90$ \\
\hline Cassette & Wafer cassette & $270^{*} 260^{*} 200$ & $40^{*} 23^{*} 18$ \\
\hline Robot arm & SMIF robot arm & $480^{*} 92^{*} 208$ & $70^{*} 8^{*} 18$ \\
\hline & & & \\
\hline
\end{tabular}

enclosure, a SMIF for controlling and transporting wafer cassette and a SMIF robot arm for transferring the product in and out of the process equipment and an empty SMIF pod at the top of SMIF enclosure. The mini-environment contains a local recirculation air supply system with velocity $v_{2}$ from a fan filter unit (FFU) to isolate product manufacturing from surrounding in order to maintain ultra clean environment. The CFX code will be used to study the flow field of air movement corresponding to the design parameters. The purpose of this article is to reduce the recirculation zone and to maintain the positive pressure from the analysis results of the airflow field and pressure distribution of SMIF enclosure and mini-environment.

\section{NUMERICAL MODEL}

The model of the actual equipment is divided into four blocks in this article, as shown in Fig. 2. Block 1 represents the region of air supply system, block 2 contains cassette and robot arm, block 3 is the empty SMIF pod, and block 4 includes the region of mini-environment. The grid choices for the entire physical domains including each block, cassette and robot arm are optimally tested. Table I shows the final optimal results of block sizes and number of cells. In the three-dimensional (3-D) model flow field, the following assumptions were made for this analysis: the flow is Newtonian and incompressible; all of the physical properties of liquid and solid material are constant; the flow is turbulence; and the thermal effect can be neglected. Using the assumptions stated above, the governing form include continuity, momentum, $k-\epsilon$ equations can be expressed as

$$
\frac{\partial(\rho \varphi)}{\partial t}+\frac{\partial\left(\rho u_{i} \varphi\right)}{\partial x_{i}}=\frac{\partial}{\partial x_{i}}\left(\Gamma_{i j} \frac{\partial \varphi}{\partial x_{j}}\right)+s_{\varphi}
$$

where $\rho$ is the air density, $\Gamma_{i j}$ is the effective diffusive coefficient, $u_{i j}$ is the air velocity vector, $S$ is the source or sink term for corresponding variable $\varphi$. The independent variable $\varphi$ includes the three velocity components $(u, v, w)$, pressure $(p)$, turbulent kinetic energy $(k)$ and the dissipation rate of turbulent kinetic energy $(\epsilon) . S \varphi$ and $\varphi$ were described by Patankar [8]. For turbulent closure the standard $k-\epsilon$ model is adopted, the five adjustable constants in $k-\epsilon$ model take the values obtained by comprehensive data fitting for a wide range of turbulent flows, which are $C_{1}=1.44, C_{2}=1.92, C_{3}=0, \sigma_{k}=1.0$ and $\sigma_{\epsilon}=1.3$.

The inlet boundary conditions at the airflow passing through the ULPA filters in the inlets of the mini-environment and SMIF enclosure assume to be uniform. The pressure in the mini-environments must be confined strictly to maintain the positive pressure state. Generally in clean room the internal pressure is $5 \mathrm{~Pa}$ larger than the outside pressure to ensure the cleanliness. For this reason, the pressure setting in all outlets are $5 \mathrm{~Pa}$ in mini-environment and SMIF enclosure. It is also assumed that on the solid surfaces no-slip conditions are required.

The above equations are solved using a commercial CFD code, CFX4, based on a finite volume discretization method. The CFD code utilizes the multi-block concept to establish a computational geometry. Within the code, the standard hybriddifferencing scheme and the semi-implicit pressure linked equation (SIMPLE) pressure correction algorithm are used in numerical analysis [9]. The CFD code checks convergence criteria by examining the residual of each solution and by the iteration times. The residual of the mass flow rate, for example, is defined as

$$
\xi_{m}=\sum_{i=1}^{n}\left|\left(m_{\mathrm{in}}-m_{\mathrm{out}}\right)_{i}\right|
$$

where $n$ is the grid number, and $m_{\mathrm{in}}$ and $m_{\text {out }}$ are the flow rates entering and leaving the control volume, respectively. In this study the residual of the mass flow rate, velocity components and turbulent kinetic energy reach $10^{-4}$ (except the turbulent dissipation). Therefore, the convergence criteria were set to be $10^{-4}$ for these residual.

\section{RESULTS AND DiSCUSSION}

In order to meet the requirement of cleanliness Class 1 or 0.1 inside the space of the SMIF enclosure, the circulation zones in the SMIF enclosure should be eliminated or reduced as much as possible, especially in the areas near the wafer cassette. To eliminate or reduce the presence of the circulation zones, the velocity gradient and pressure gradient in the flow field must be minimized as much as possible [2]. That is, the flow field 


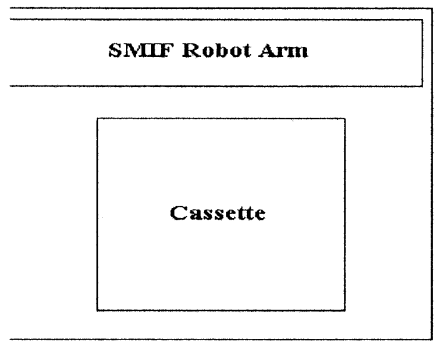

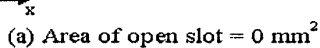

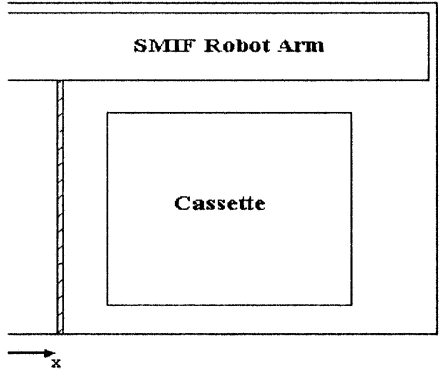

(c) Area of open slot $=2374 \mathrm{~mm}^{2}$

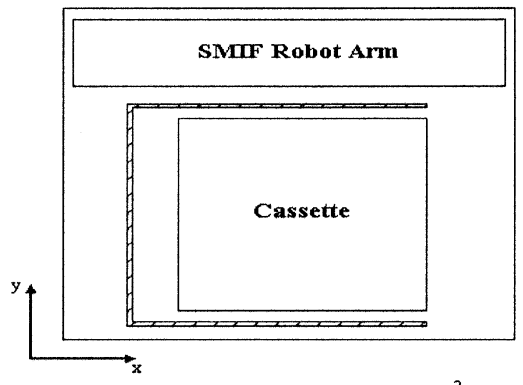

(b)Area of open slot $=8805 \mathrm{~mm}^{2}$

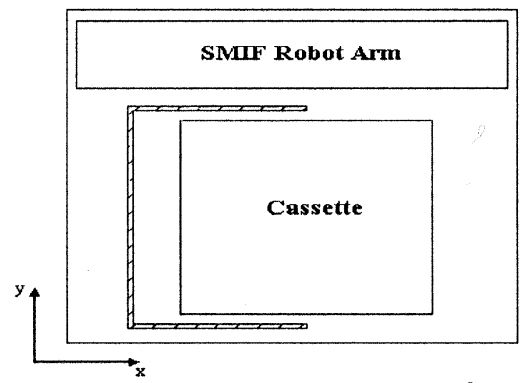

(d) Area of open slot $=5743 \mathrm{~mm}^{2}$

Fig. 3. Different positions and area of open slot at SMIF enclosure at cross section A-A in Fig. 1.
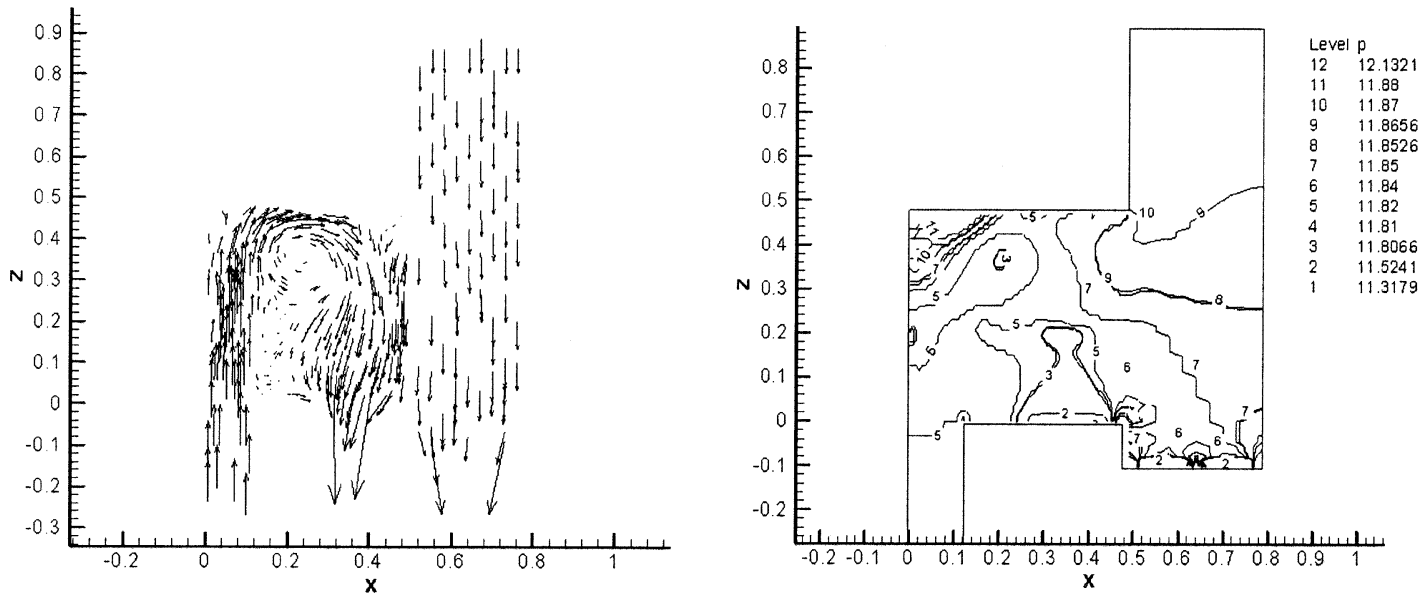

Fig. 4. Velocity and pressure distributions at position and area on $y=0.322 \mathrm{~m}$.

should approach uniformity. Besides, the gauge pressure must be strictly maintained $10 \mathrm{~Pa}-17 \mathrm{~Pa}$ in mini-environment and SMIF enclosure.

The major design parameters affecting the flow field include the position and size of slot drilling at the bottom of the SMIF enclosure, inlet air velocity of the SMIF enclosure $\left(v_{1}\right)$, inlet air velocity of the mini-environment $\left(v_{2}\right)$, the leakage area on the contact face between the mini-environment and SMIF enclosure and the position of the robot arm in the SMIF enclosure. The effect of above parameters on the flow fields and pressure distributions are discussed in the following sections.

Slotting at the bottom of the SMIF enclosure and changing the air flow velocity inside the SMIF enclosure can be adopted to obtain better flow field distribution and eliminate or reduce the presence of circulation zones [3], [5]. Fig. 3 shows four different models with regard to different slotting open position and area at the cross section A-A in Fig. 1, which locates at the SMIF equipment. Slotting at this location can be easily manufactured and effectively improved the flow field and reduced the unwanted recirculation area. Four different slot open positions and area can provide the information of their impact on the flow field. Except for differences in the position and area of slot, the rest of the boundary conditions are the same in all cases. In order to supply uniform flow in mini-environment, the slot porosity at the air outlet of the mini-environment is set $12 \%$. Besides, the velocity at the SMIF air inlet is set at $0.5 \mathrm{~m} / \mathrm{s}$ and that at the mini-environment air inlet $0.3 \mathrm{~m} / \mathrm{s}$.

The flow field of the simulation result is recorded based on the flow channel ( $y=0.322$ plane) between the wafer cassette and transport robot mainly because the information obtained on the plane is more useful and easier to analyze. Fig. 4 shows the velocity and pressure distributions for the $5473 \mathrm{~mm}^{2}$ case. The 


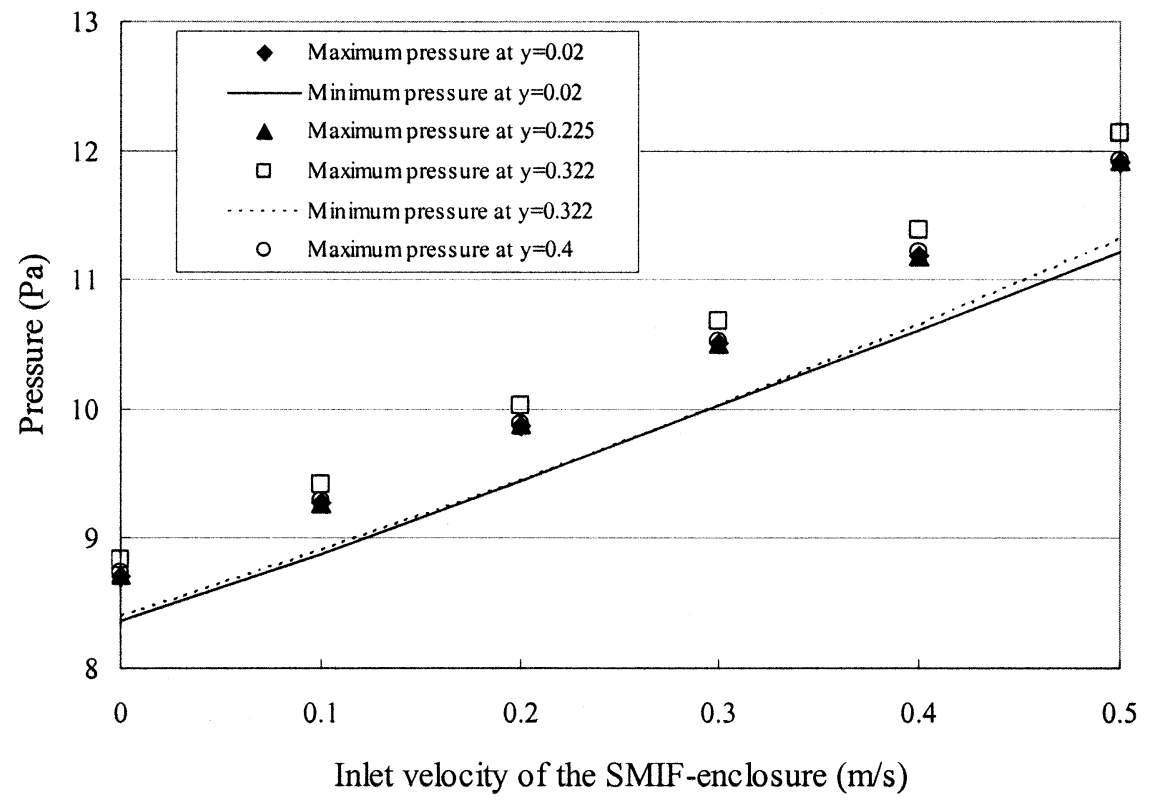

Fig. 5. Dependents of the pressure variations on different positions and inlet velocity of the SMIF enclosure.
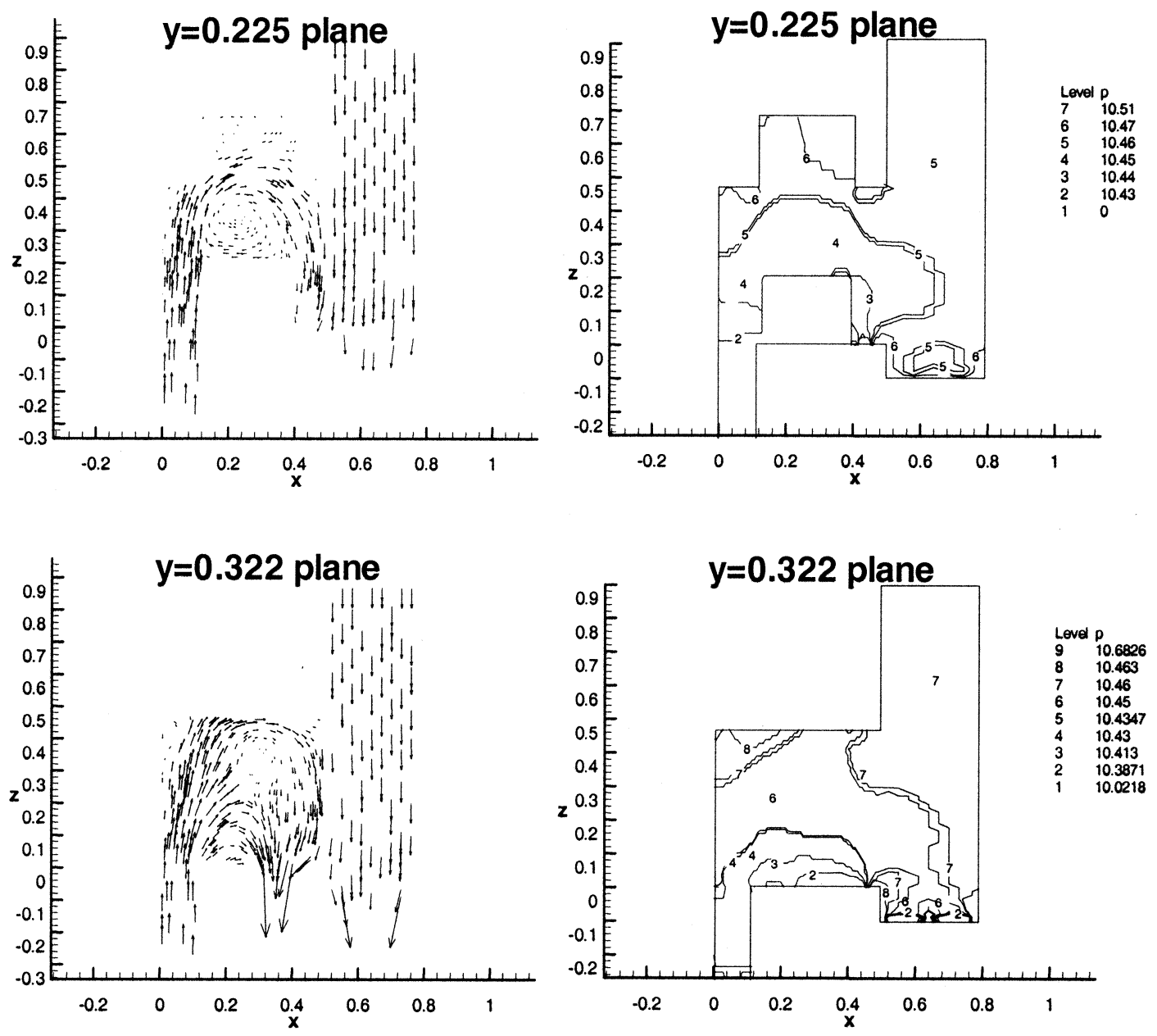

Fig. 6. Velocity and pressure distributions at the SMIF enclosure under $v_{1}=0.3 \mathrm{~m} / \mathrm{s}$ on $y=0.225 \mathrm{~m}$ and $y=0.322 \mathrm{~m}$.

flow field does not exist, which is obvious in the circulation zones. In addition, the pressure distribution also maintains the positive pressure and conforms to the requirement range inside
SMIF enclosure. Thus, the bottom slot open in all of the following numerical simulation cases is based on the design. Only the other design parameters vary. 


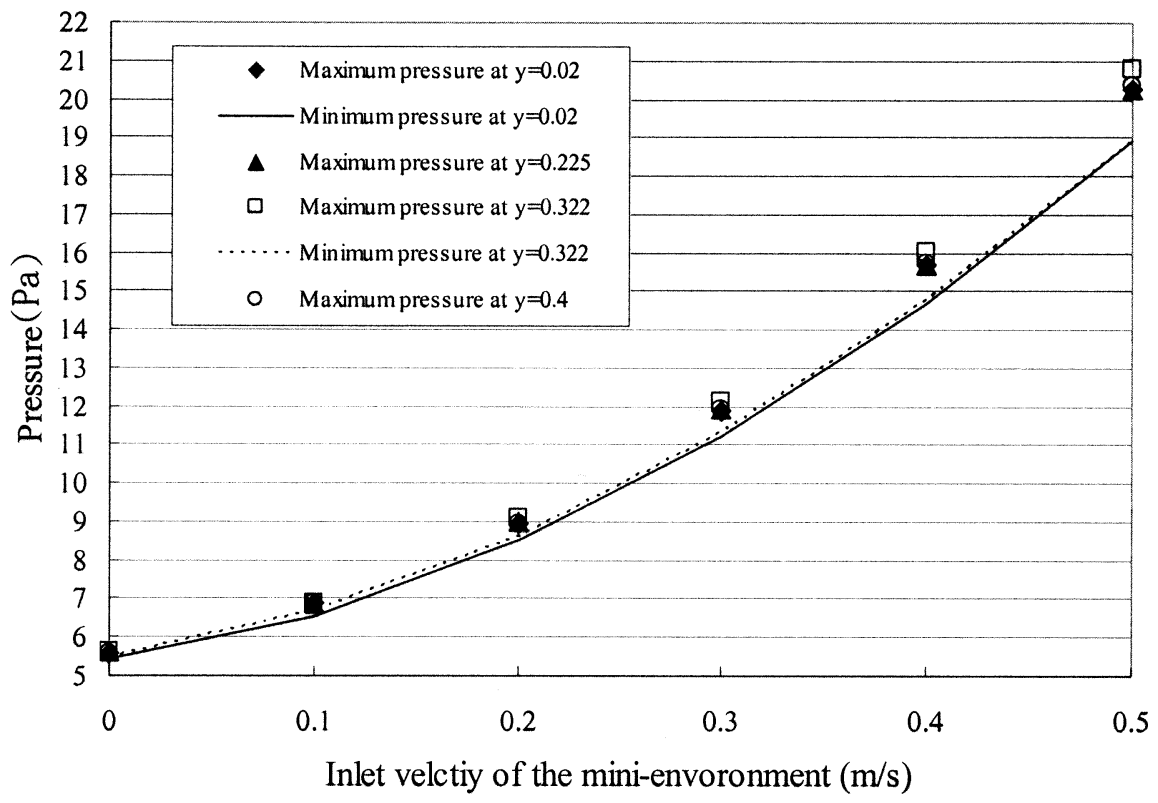

Fig. 7. Dependents of the pressure variations on different positions and inlet velocities of the mini-environment.
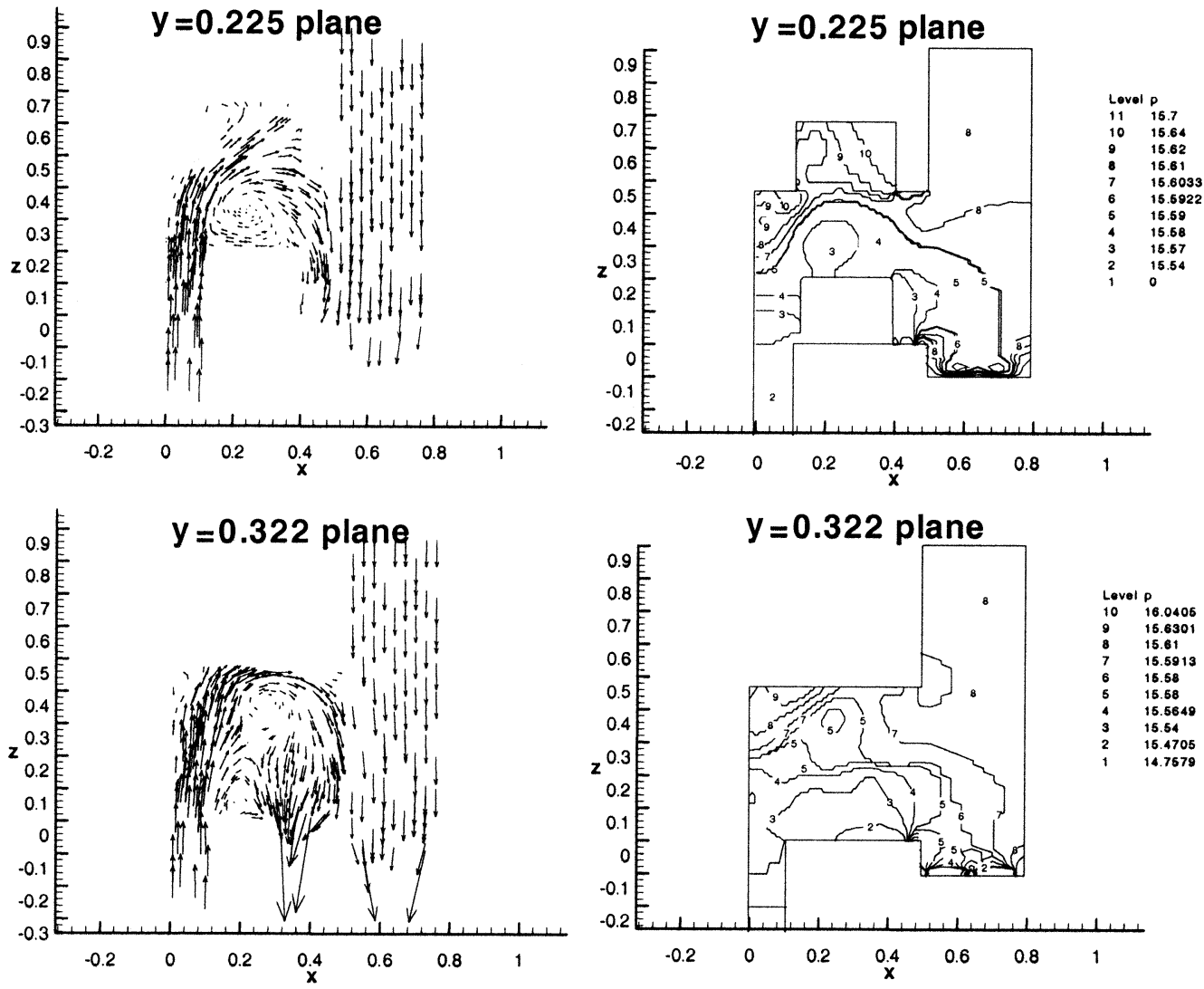

Fig. 8. Velocity and pressure distributions at the SMIF enclosure under $v_{2}=0.4 \mathrm{~m} / \mathrm{s}$ on $y=0.225 \mathrm{~m}$ and $y=0.322 \mathrm{~m}$.

The velocity difference between the influx airflow and zone airflow should be minimized to increase the cleanliness [4]. Here, different inlet velocities $\left(v_{1}\right)$ at SMIF enclosure and minienvironment are simulated to analyze the correlation between the above velocity and the circulation zones to serve as a reference for the optimization SMIF design. Since the maximum supply of airflow velocity by the FFU (fan filter unit) cannot exceed $0.5 \mathrm{~m} / \mathrm{s}$, the maximum velocity of the simulations cases must be $0.5 \mathrm{~m} / \mathrm{s}$. Then, the inlet velocities $\left(v_{1}\right)$ of SMIF enclosure and mini-environment are set at $0-0.5 \mathrm{~m} / \mathrm{s}$, and the inlet velocity at the mini-environment and SMIF enclosures are chosen to be $0.3 \mathrm{~m} / \mathrm{s}$ and $0.5 \mathrm{~m} / \mathrm{s}$, respectively.

The numerical results are shown in Figs. 5-8. In Fig. 5, at $v_{2}=0.3 \mathrm{~m} / \mathrm{s}$, to meet the requirement of the positive pressure 


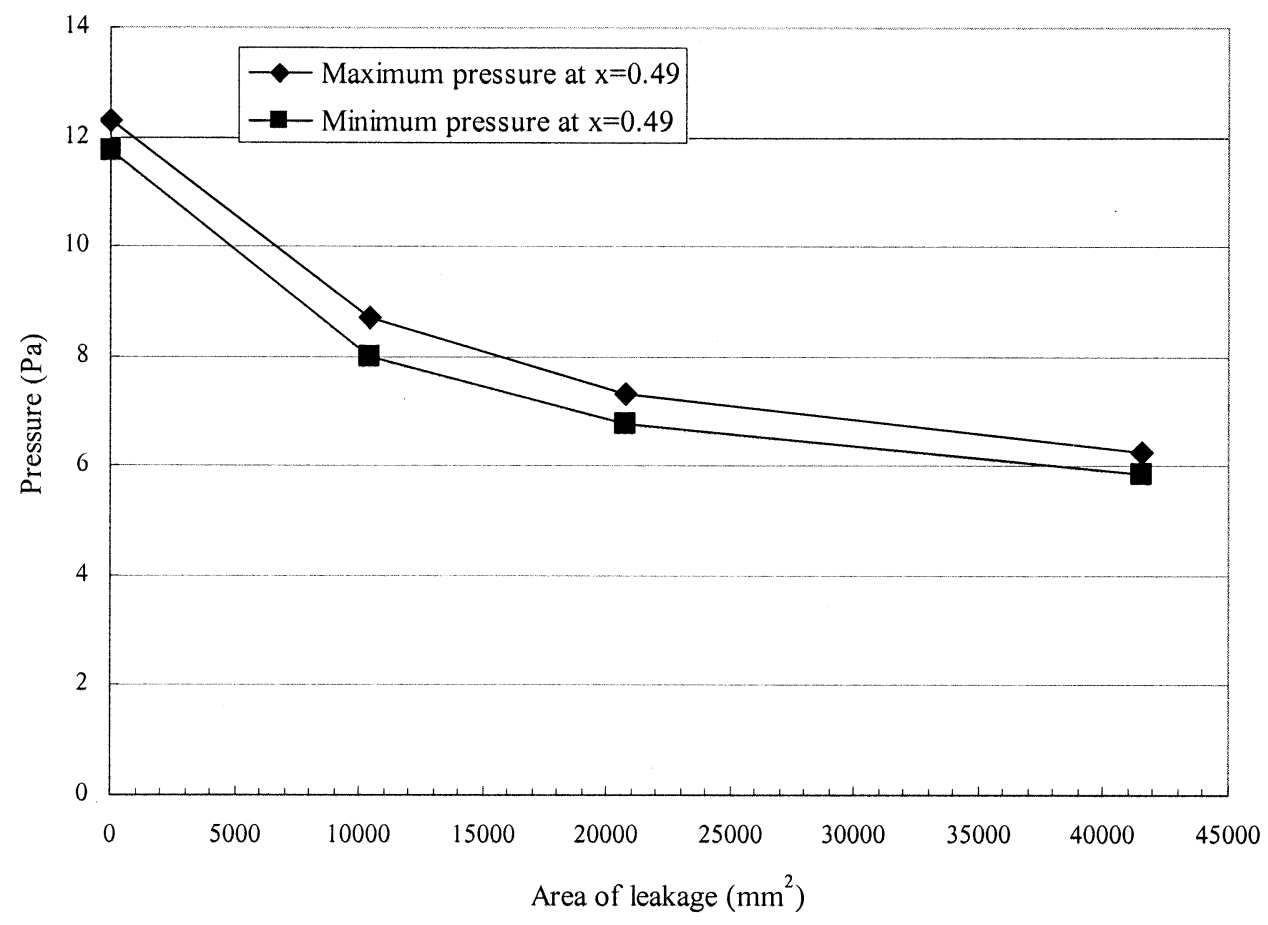

Fig. 9. Dependents of the pressure variations on different leakage cases.
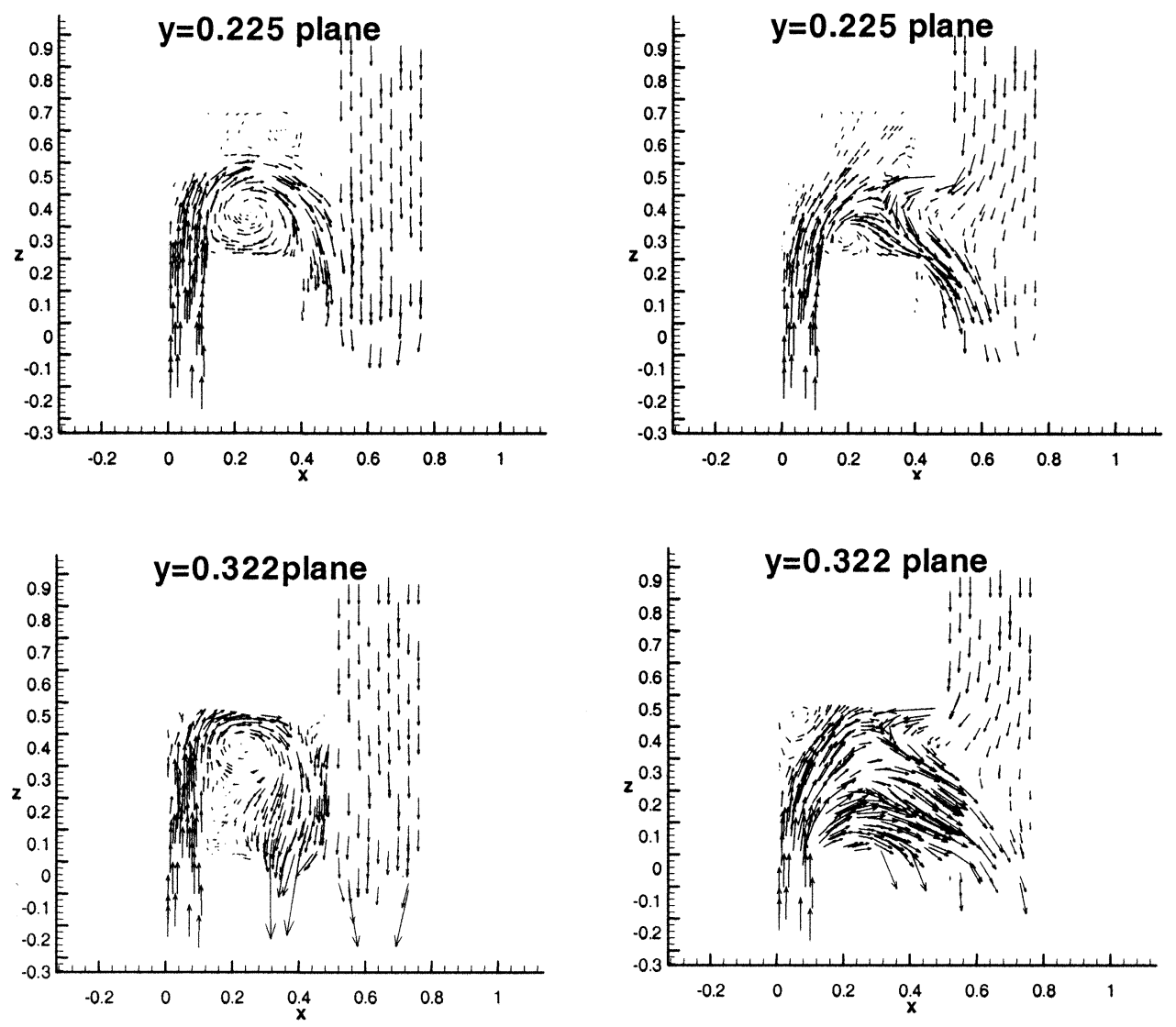

Fig. 10. Velocity distributions under area of $=0$ and $20773 \mathrm{~mm}^{2}$.

difference of $5 \mathrm{~Pa}-12 \mathrm{~Pa}$, the value of $v_{1}$ must reach at least $0.3 \mathrm{~m} / \mathrm{s} . v_{1}=0.3,0.4$, and $0.5 \mathrm{~m} / \mathrm{s}$ all meet this requirement. As shown in Fig. 6, the pressure distribution of $0.3 \mathrm{~m} / \mathrm{s}$ displays a better uniformity and no low pressure zone. And the airflow obviously flows toward the slotting at the bottom. As shown in Fig. 7, to meet the requirement of the positive pressure, when $v_{1}=0.5 / \mathrm{s}$, the $v_{2}$ value ranges between $0.25 \mathrm{~m} / \mathrm{s}$ and $0.42 \mathrm{~m} / \mathrm{s}$. Fig. 8 shows the velocity and pressure distributions at $v_{2}=$ 

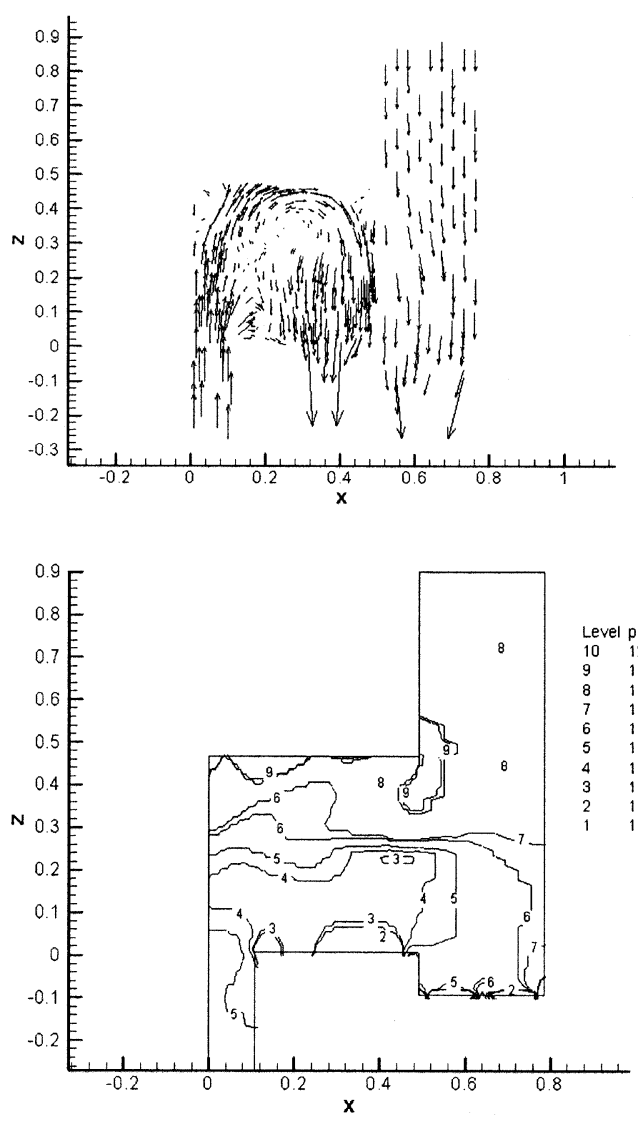

Position 1
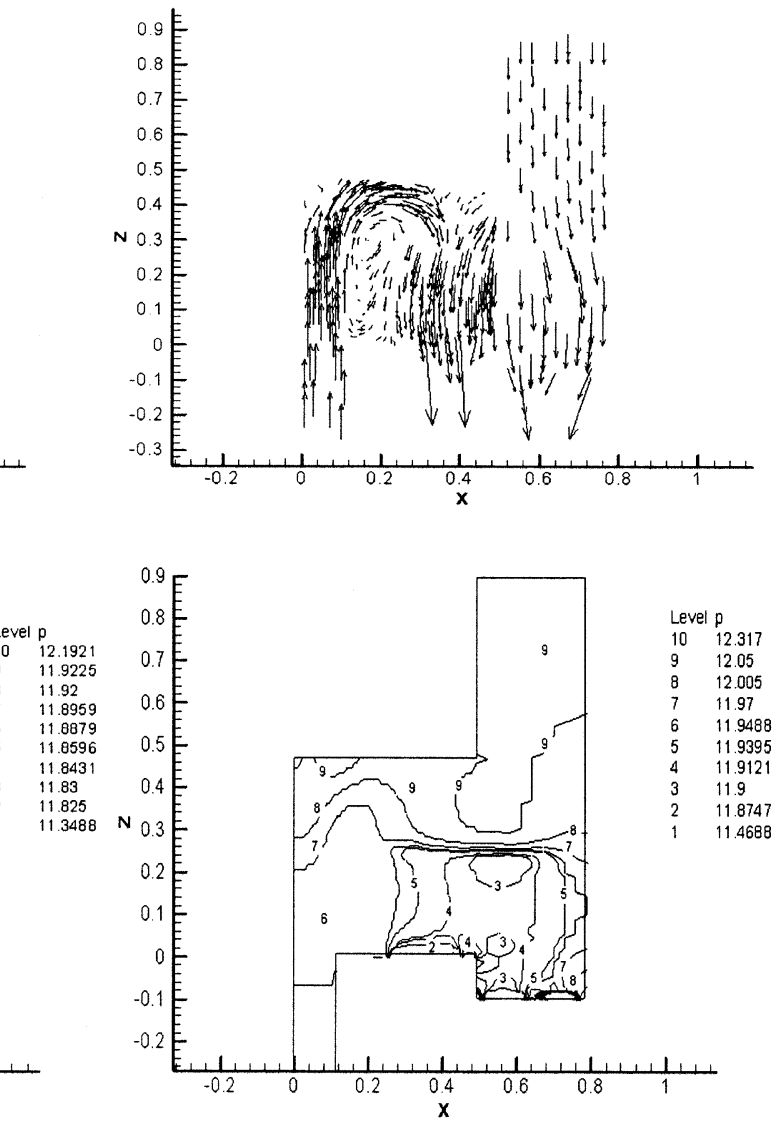

Position 2

Fig. 11. Velocity and pressure distributions under different robot positions.

$0.4 \mathrm{~m} / \mathrm{s}$. The circulation zone tends to decrease. This trend is even more pronounced when examined by the pressure. In fact, the change of velocity of the mini-environment does not have a significant impact on the flow field in SMIF. The effect of the changes in inlet velocity falls mainly on the pressure field. The reason is that the inlet cross section of the mini-environment is around 4.58 times of the SMIF enclosure inlet, producing a greater impact on the pressure field. Besides, since the airflow of SMIF enclosure comes primarily from the enclosure inlet, the flow pattern should naturally sustain a greater impact.

The area of leakage between SMIF enclosure and mini-environment for the simulation cases chosen to be $0,10387,20773$, and $41547 \mathrm{~mm}^{2}$, and the results shown in Figs. 9 and 10. In Fig. 9, the findings indicate that the larger the leakage area between SMIF and the mini-environment, the lower the pressure and the more difficult it is to maintain the positive pressure. In addition, the requirement of a positive pressure different of at least $5 \mathrm{~Pa}$, when $v_{1}=0.5 \mathrm{~m} / \mathrm{s}$ and $v_{2}=0.3 \mathrm{~m} / \mathrm{s}$, the leakage area must not exceed $5000 \mathrm{~mm}^{2}$. If the leakage area exceeds $5000 \mathrm{~mm}^{2}$, the inlet velocity $v_{2}$ must exceed $0.3 \mathrm{~m} / \mathrm{s}$. Comparing two different leakage area 0 and $20773 \mathrm{~mm}^{2}$, the Fig. 10 indicate that the flow field of the case of larger leakage area displays only a small circulation zone at the upper left corner in the SMIF enclosure for the largest leakage area. But the greater the effect of the airflow from the SMIF enclosure on the flow field in the mini-environment. Therefore, to adjust the leakage area
TABLE II

Positions of THE WAFER CASSETTE AND RoBot ARM

Position $1 \quad$ Position 2

\begin{tabular}{lll}
\hline Distance of horizontal moving (x-direction), & 0.056 & 0.055 \\
$\mathrm{~mm}$ & & \\
\hline Distance of vertical moving (z-direction), mm & 0.102 & 0.104 \\
\hline
\end{tabular}

between SMIF and the mini-environment can effectively eliminate the circulation zone within the pressure requirement.

The movement of the robot arm between SMIF and the minienvironment certainly affects the flow field. Here, the positions of the wafer cassette and robot arm are shown in Table II. The inlet velocity of the SMIF enclosure and the inlet velocity of the mini-environment are set at 0.5 and $0.3 \mathrm{~m} / \mathrm{s}$ respectively. Fig. 11 shows the velocity vector plot. The flow pattern is not affected by the wafer cassette and robot arm positions. Thus, only a larger circulation zone is found in position 1 . However, as the wafer cassette and robot moves to the right, the overall internal pressure value tends to increase in pressure-contour diagram. The reason is that part of the wafer cassette and robot arm, after entering the mini-environment, blocks the downward 
airflow at the mini-environment inlet, which raises the pressure in the flow field.

\section{CONCLUSION}

To achieve the requirement of cleanliness in the mini-environment and SMIF enclosure, the present CFD simulation results for flow field and pressure distribution are shown for different parameters. The effects of major parameter are summarized below.

1) Slotting at the bottom of the SMIF enclosure strongly influences the flow pattern and pressure distribution. Position and area of slot are appropriately set to reduce the circulation zone and to solve the requirement of positive pressure effectively.

2) As the air inlet velocity of the SMIF enclosure reaches $0.3 \mathrm{~m} / \mathrm{s}$ to meet the requirement of the positive pressure, the value of the inlet velocity of the mini-environment must maintain at least $0.3 \mathrm{~m} / \mathrm{s}$. Additionally, when inlet velocity of the SMIF enclosure equals $0.5 \mathrm{~m} / \mathrm{s}$, the inlet velocity of the mini-environment ranges between $0.25 \mathrm{~m} / \mathrm{s}$ and $0.42 \mathrm{~m} / \mathrm{s}$. The air inlet velocity of the SMIF enclosure affects the flow pattern more than that of the mini-environment.

3) The larger leakage rate between SMIF enclosure and the mini-environment can land to the greater effect of the airflow from the mini-environment on the flow field in the SMIF enclosure. The larger leakage rate brings on the lower pressure and consequently it is more difficult to maintain the positive pressure.

4) As the wafer cassette and robot arm moves into the mini-environment, the overall internal pressure value tends to increase.

\section{REFERENCES}

[1] M. Parikh and Kaempf, "SMIF: A technology for wafer cassette transfer in VLSI manufacturing," Solid State Technol.,pp. 110-115, July 1984

[2] T. Yamamoto, A. S. Viner, R. P. Donovan, and D. S. Ensor, "Modwl study of contaminant flow in the vicinity of semiconductor processing equipment," J. IES , pp. 19-24, July/Aug. 1990.

[3] W. Scheler, P. Vorberg, H. Schneider, and P. Fabian, "SMIF integration of a wafer prober by components of jenoptik GmbH," Proc. Inst. Environmental Sci., pp. 211-217, 1995.

[4] G. L. Quarini and Y. C. Chang, "Experimental measurements and theoretical predictions of the thermohydraulic performance of clean rooms for the semi-conductor industry," J. Process Mech. Eng., vol. 210, pp. $9-18,1996$.

[5] A. G. Tannous, "Air flow simulation in a minienvironment," Solid State Technol., pp. 201-209, July 1996.

[6] J. Milberg, J. Fischbacher, and A. Engel, "Fluidic integration of equipment in cleanrooms," Solid State Technol., pp. 43-45, August 1991.

[7] J. Schliesser, "Designing methods to obtain a less contaminated air-flow at the product level," Proc. Inst. Environmental Sci., pp. 406-412, 1994
[8] S. V. Patankar, Numerical Heat Transfer and Fluid Flow. New York: McGraw-Hill, 1980.

[9] CFX 4.2 Flow Solver User Guide, AEA Technology, 1997.

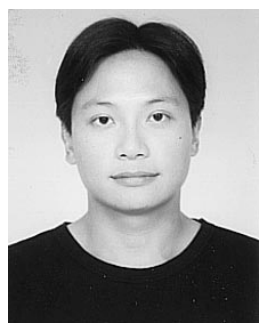

Huan-Ruei Shiu was born in Penghu, Taiwan, ROC, in 1974. He graduated from the Department of Marine Engineering, National Kaohsiung Institute Technology in 1994. He received the B.Sc. degree in marine engineering and technology from the National Taiwan Ocean University in 1996, the M.Sc. degree in mechanical engineering from Tatung University in 1998. He is working toward the Ph.D. degree in mechanical engineering from the National Taiwan University, Taipei, Taiwan, R.O.C.

His current research interests include CFD, HVAC system of cleanroom, semiconductor engineering, and MEMS design.

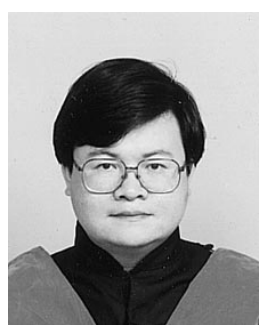

Hsiao-Yi Huang was born in Taipei, Taiwan, R.O.C., in 1969. He received the M.Sc. degree in naval architecture and ocean engineering from National Taiwan University, Taipei, Taiwan, R.O.C., in 1997. He is working toward the Ph.D. degree in mechanical engineering from the National Taiwan University.

His current research interests include HVAC system supervisory control, semiconductor engineering, and MEMS design.

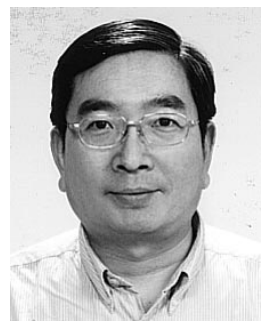

Sih-Li Chen was born in Nantou, Taiwan, R.O.C., in 1956. He received the B.Sc. and M.Sc. degrees from National Taiwan University, Taipei, Taiwan, R.O.C., in 1979 and 1981, respectively, all in mechanical engineering. In 1987, he received the Ph.D. degree from the University of California, Berkeley.

Since 1987, he has been with the Department of Mechanical Engineering, National Taiwan University, where he was a Professor. In 1999, he was chairman of ASHRE in Taiwan. His current research interests include HVAC system in building, cleanroom design, and semiconductor factory process exhaust system.

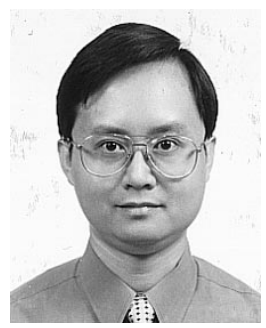

Ming-Tsun Ke was born in Chiayi, Taiwan, R.O.C., in 1966. He received the B.Sc., M.Sc. and the Ph.D. degrees from National Taipei University of Technology, Taipei, Taiwan, R.O.C., in 1988, 1990, and 1994, respectively, all in mechanical engineering.

$\mathrm{He}$ is an Associate Professor in Department of Air-Conditioning and Refrigeration engineering, National Taiwan University. His current research interests include energy saving in HVAC system in building, cleanroom design, and semiconductor factory process exhaust system. 